\title{
Analytical modelling of intragranular backstresses due to deformation induced dislocation microstructures
}

\author{
M. Sauzay* \\ CEA Saclay, DEN-DMN-SRMA, Bâ timent 455, 91191 Gif-Sur-Yvette Cedex, France
}

\begin{abstract}
Deformation induced dislocation microstructures appear in Face-Centred Cubic metals and alloys if applying large enough tensile/cyclic strain. These microstructures are composed of a soft phase with a low dislocation density (cell interiors, channels...) and a hard phase with a high dislocation density (walls). It is well known that these dislocation microstructures induce backstresses, which give kinematic hardening at the macroscopic scale. A simple two-phase localization rule is applied for computing these intragranular backstresses. This is based on Eshelby's inclusion problem and the Berveiller-Zaoui approach. It takes into account an accommodation factor. Close-form formulae are given and permit the straightforward computation of reasonable backstress values even for large plastic strains. Predicted backstress values are compared to a number of backstress experimental measurements on single crystals. The agreement of the model with experiments is encouraging. This physical intragranular kinematic hardening model can easily be implemented in a polycrystalline homogenization code or in a crystalline finite element code. Finally, the model is discussed with respect to the possible plastic glide in walls and the use of enhanced three phase localization models.
\end{abstract}

Keywords: Crystal plasticity; Backstress; Metallic material; Microstructures; Dislocations

\footnotetext{
* Tel.: +33 1690835 67; fax: +33 169087167.

E-mail address: maxime.sauzay@cea.fr
} 


\section{Introduction}

Monotonic and cyclic behaviours of Faced Cubic Centred (FCC) single crystals and polycrystals have been extensively studied. Numerous Transmission Electronic Microscopy (TEM) studies have shown that deformation induced dislocation microstructures appear in Faced Cubic Centred metals and alloys if the tensile/cyclic applied strain is large enough (Lukas et al., 1968; Mughrabi, 1987; Polak, 1991; Gerland et al., 1989; Mughrabi, 1983) (Fig. 1). Other crystallographic structures can be concerned too. The dislocation microstructures are composed of a soft phase (cell interiors, channels) with a low dislocation density ( $\rho_{\mathrm{c}}<10^{14} \mathrm{~m}^{-2}$, often mobile dislocations) and a hard phase (walls) with a high dislocation density ( $\rho_{\mathrm{w}}>10^{15} \mathrm{~m}^{-2}$, often edge dipoles) (Mughrabi, 1987). Depending on the crystallographic orientation with respect to the solicitation axes, loading type (cyclic/monotonic) and strain level, walls/channels, cells or labyrinths have been observed (Fig. 1). In single crystals containing such dislocation microstructures, backstresses have been measured using different kinds of procedures (Mughrabi, 1983). Direct mechanical measures using the hysteresis loops (Cottrell, 1953; Kuhlmann-Wilsdorf and Laird, 1979), TEM dislocation radii measures (Mughrabi, 1987) and X-rays diffraction (Mughrabi et al., 1986; Ungar et al., 1991) have been used. These backstresses are of opposite signs in the soft and hard phase. They are due to the incompatibilities in the single crystal (dislocations glide easily in the soft phase, but hardly in the hard phase). They induce kinematic hardening at the macroscopic scale. Such deformation dislocation microstructures appear also in polycrystals. They depend partially on the grain orientations with respect to the solicitation axes (Winter et al., 1981; Polak et al., 1992; Hansen and Huang, 1998). Backstresses at the micrometer scale have been measured by synchrotron radiation (Holden et al., 1998), but they are due to both intragranular and intergranular plastic heterogeneities, and not only to intragranular ones.

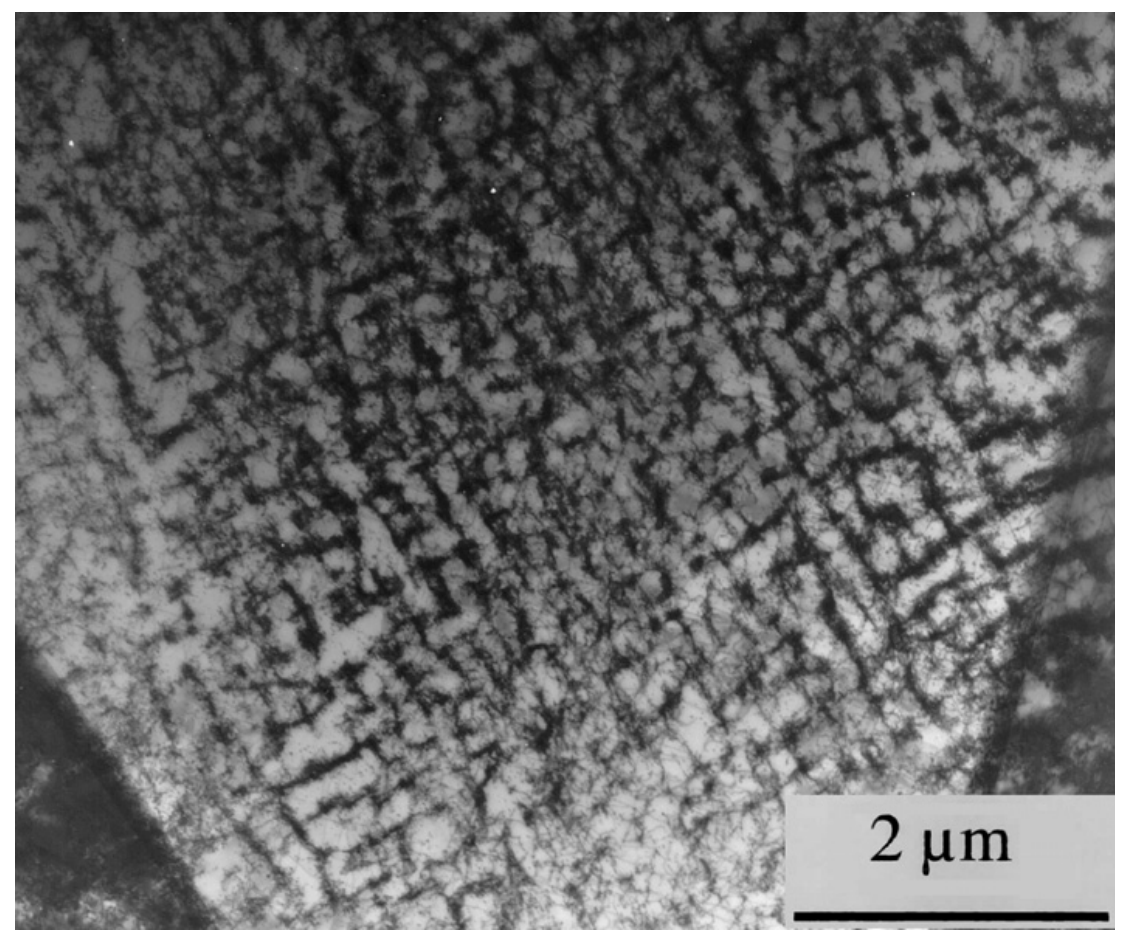

Fig. 1. Example of a dislocation microstructure in an austenitic steel (labyrinth microstructure). Cyclic alternated stress, $\Delta \varepsilon=0.6 \%, \mathrm{~d} \varepsilon / \mathrm{d} t=3 \times 10^{-3} \mathrm{~s}^{-1}, T=550{ }^{\circ} \mathrm{C}$ (I. Monnet, SRMA). 
Concerning computations, there are at least three kinds of dislocation microstructure backstress computation models have been proposed in the past. First, several models based on inclusion problems have been proposed. They consider the soft phase as surrounded by the matrix or by an elastic wall and by the matrix. They use hypotheses concerning the localization rule which links the macroscopic scale to the cell or wall/channel scale. Mughrabi's model supposes homogeneous strain (Voigt's model) (Mughrabi, 1983), Pedersen's one uses Eshelby's transition rule (Eshelby, 1957; Kröner, 1961; Pedersen, 1990) and the Lemoine et al. one considers a cell interior surrounded by a wall and embedded in a matrix (Fig. 2) (Lemoine et al., 1994; Langlois and Berveiller, 2003). Lemoine et al. model permits us to take into account the geometry and the wall volume fraction which characterize the dislocation microstructure. Generally, the walls are supposed to be elastic, the soft phase is elastoplastic and the matrix is elastic or elastoplastic. The soft phase backstress depends on the soft phase plastic slip. Using Eshelby's coefficients (Eshelby, 1957), it is possible to compute the 3D backstress tensor induced by a given microstructure geometry and by the plastic slips given on several slip systems. But, all these models are thermoelastic and use no plastic accommodation factor; that is why the computed backstresses are too high as soon as the plastic strain is higher than a few $10^{-4}$ (Berveiller and Zaoui, 1979; Sauzay, 2000). In fact, thermoelastic models suppose homogeneous strains in each phase even if there are heterogeneities in each phase, particularly near the interfaces. These heterogeneities permit the accommodation of the deformation and reduce the incompatibilities and backstresses. That is why an accommodation factor classically used in polycrystalline models (such as Berveiller and Zaoui model (1979)) has been used in this work.

There are at least two kinds of other backstress models. Mughrabi proposes a second model based on the hypothesis that both the hard and soft phases are plastically deformed at maximal load (Mughrabi, 1987). This has been developed more recently by Feaugas and Gaudin (2001). The computed soft phase backstress depends on the dislocation densities in the two phases but not directly on the plastic slip. Another model has been proposed by Harder (1999). This one is more phenomenological and uses adjustable parameters like macroscopic kinematic hardening models (Lemaitre and Chaboche, 1987). These two models do not take into account the dislocation microstructure geometry (walls/channels,

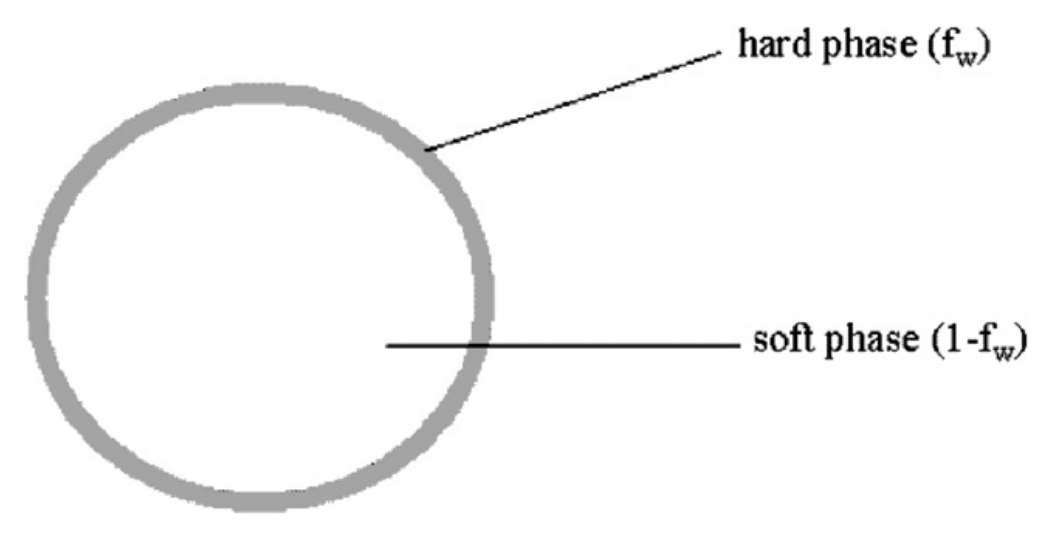

matrix

Fig. 2. Equiaxed dislocation cell (tensile or high cyclic plastic strain). 
equiaxed cells). These models permit us to compute the value of the backstress on a slip system. But, the 3D backstress tensor computation is not so clear in spite of the proposition of Harder (1999) because of lack of a physical base. Homogenization codes (Cailletaud, 1992; Langlois and Berveiller, 2003) as well as crystalline finite element codes (Barbe et al., 2001; Clayton and McDowell, 2003; Erieau and Rey, 2004; Bertolino et al., 2005; Delannay et al., 2006; Nakamachi et al., 2007) are more and more used. Even when they take into account intragranular kinematic hardening laws, they generally use at the grain scale macroscopic type laws and several adjustable parameters without any clear physical meaning. Recently several enhanced models were proposed for simulating forming processes on large structures (Tugcu et al., 2004; Habraken and Duchêne, 2004; Raabe and Roters, 2004; Erieau and Rey, 2004; Guan et al., 2006). But they did not consider any intragranular kinematic hardening model even if dislocation microstructures are usually observed if large plastic strains are applied. This could reduce their prediction ability even if there is a huge development of these micromechanical models and of their scale transition laws (enhanced homogenization modelling, comparisons between homogenization and finite element code results at both grain and macroscopic levels). Kinematic hardening based on deformation induced macrostructure should be particularly important for modelling strain path changes during which dislocation microstructures evolve as mentioned by Langlois and Berveiller (2003) or Beyerlein and Tomé (2007). Recently, discrete dislocation dynamics (DDD) was used for evaluating the long-range stress field induced by particular dislocation microstructures (Khan et al., 2004; Déprés, 2004). But, DDD requires time-consuming numerical computations and does not give close-form expressions which can be directly implemented in crystalline finite element codes.

Experimental in situ TEM observations of Tabata et al. $(1977,1982)$ show that the plastic glide in the walls is much reduced. Tabata et al. study [1 111$]$ single crystals (Tabata et al., 1977). They show that the slip lines stop at the cell walls and do not cross them (Tabata et al., 1977) and that the dislocations glide much faster in the middle of the cells than near the walls (Tabata et al., 1982). These observations permit an experimental evaluation of the dislocation speeds which are larger in the middle of the cells. The dislocation densities are so high in the walls that the dislocation motion through them seems to be limited.

Because of these experimental results, elastic walls and elastoplastic soft phase have been considered in this work. An inclusion approach has been used in order to take into account the dislocation microstructure geometry (except the enrobed character). Following the Berveiller-Zaoui approach, a two-phase model is applied. The constitutive law of the whole single crystal is supposed to be isotropic elastoplasticity. The average secant modulus and finally the Berveiller-Zaoui accommodation factor are used. In spite of the crystalline behaviour anisotropy, the used localization rule supposes isotropic behaviour in order to propose close-form formulae. The predicted (soft phase) backstresses are compared with many backstress measures in single crystals for which the backstresses are reduced to the intragranular ones. Even for large strains, the computations agree rather well with the experimental measures. This physical intragranular kinematic hardening model can be easily be implemented in a polycrystalline homogenization code or in a crystalline finite element code. These results are finally discussed, particularly concerning possible plastic glide inside walls (which has been neglected in a first attempt) and predictions of enhanced three phase inclusion-matrix models (which take into account the enrobed character of the soft or hard phase but do not give any close-form expression). 


\section{Intragranular backstress computation}

Because of their high dislocation densities, the walls are supposed to be elastic, whereas the soft phase is elastoplastic. For the sake of simplicity, a single crystal is considered. Let us consider a spherical cell (Fig. 2). This two-phase cell is supposed to be embedded in an infinite matrix which constitutive law is the whole single crystal one. Crystalline plasticity is considered. Therefore, the average plastic strain tensor is decomposed into a sum of plastic shear tensors. The average plastic slip is denoted as $\gamma_{i}^{\mathrm{p}}$ and $n_{i}, m_{i}$ are respectively the slip normal and slip direction of the corresponding $i$ th slip system. There are $N$ slip systems $(N=12$ for the FCC structure and $N=24$ for the Body Cubic Centred structure). In the framework of small-strain theory, the whole single crystal plastic strain tensor is given by:

$$
\left.\underline{\underline{\varepsilon^{\mathrm{p}}}}=\sum_{i=1}^{N} \gamma_{i}^{\mathrm{p}} \frac{1}{2} \underline{\left(n_{i}\right.} \underline{m_{i}^{\mathrm{T}}}+\underline{m_{i}} \underline{n_{i}^{\mathrm{T}}}\right)
$$

As walls are supposed to be elastic, plastic deformation is localized in the soft phase only:

$$
\underline{\underline{\varepsilon^{\mathrm{p}}}}=\left(1-f_{\mathrm{w}}\right) \underline{\underline{\varepsilon_{\mathrm{s}}^{\mathrm{p}}}}
$$

with $f_{\mathrm{w}}$ the wall volume fraction and using the soft phase plastic strain tensor.

Following Kröner's thermoelastic approach applied to a spherical inclusion (Kröner, 1961), the localization rule gives the soft phase backstress tensor:

$$
\underline{\underline{x_{\mathrm{c}}}}=-2 \mu(1-\beta)\left(\underline{\underline{\varepsilon_{\mathrm{s}}^{\mathrm{p}}}}-\underline{\underline{\varepsilon^{\mathrm{p}}}}\right)
$$

with $\mu$ the elastic shear modulus, $\beta=2(4-5 v) / 15(1-v)$ (Eshelby's analysis for a spherical inclusion) (Eshelby, 1957; Mura, 1987), $v$ the Poisson ratio. This corresponds to the backstress acting inside the cell.

Using Eqs. (1)-(3), Lemoine et al. (1994) obtain the soft phase backstress for a spherical cell:

$$
\underline{\underline{x_{\mathrm{c}}}}=-\frac{f_{\mathrm{w}}}{1-f_{\mathrm{w}}} \mu(1-\beta) \sum_{i=1}^{N} \gamma_{i}^{\mathrm{p}}\left(\underline{n_{i}} \underline{m_{i}^{\mathrm{T}}}+\underline{m_{i}} \underline{n_{i}^{\mathrm{T}}}\right)
$$

Following Kröner's analysis (Kröner, 1961), this formula supposes elastic interactions between the matrix and the two-phase cell (Langlois and Berveiller, 2003; Mura, 1987).

But, it is well known that a plastic accommodation takes place for large plastic strains, which decreases the backstress level: Eq. (4) can overestimate the backstress level (Berveiller and Zaoui, 1979; Sauzay, 2000). The comparison between the predictions of Eq. (4) and the experimental backstresses measures will be discussed in Part 4. It will be shown that Eq. (4) overestimates largely the backstresses (see Tables 1 and 2). Physically, there are heterogeneous plastic strains near the interfaces between the three media, which induces backstress accommodation. In order to take this effect into account, a classical localization rule proposed by Berveiller and Zaoui (1979) is used which introduces the elastoplastic secant moduli and an accommodation factor. Such approach is suggested by Langlois and Berveiller (2003) who obtain too high backstresses using the previous Kröner approach. The Berveiller and Zaoui approach was initially proposed for studying the interaction between one grain and the whole polycrystal. It is now applied to a twophase material (hard/soft phase). In order to introduce plastic accommodation, a simple 
Table 1

Application of the Berveiller-Zaoui model to a two phases composite (Eq. (2))

\begin{tabular}{lllllll}
\hline$\gamma^{\mathrm{p}}(\%)$ & $\tau$ & $f_{\mathrm{w}}$ & $x_{\mathrm{c}}(\exp )$ & $x_{\mathrm{c}}(\mathrm{B}-\mathrm{Z})$ & $x_{\mathrm{c}}$ (Kröner) & Stress measurement \\
\hline $\begin{array}{l}\text { Copper, well-oriented } \\
38.2\end{array} \quad 44$ & $0.22( \pm 15 \%)$ & $26( \pm 20 \%)$ & $31.1( \pm 18 \%)$ & $\approx 5700$ & TEM \\
$\begin{array}{l}\text { Copper, }\langle 001\rangle \text { loading axes } \\
104\end{array} \quad 75$ & $0.3( \pm 10 \%)$ & $8.6( \pm 15 \%)$ & $9.5( \pm 15 \%)$ & $\approx 2500$ & X-ray diffraction \\
\hline
\end{tabular}

Comparison experiment/model. Tensile loading, large plastic strains. Stress unit is MPa. Geometry: equiaxed cell. Experiment references: Essmann (1965), Fourie (1968), Mughrabi et al. (1986), and Ungar et al. (1991). Amplitudes of the backstresses are given.

Table 2

Application of the Berveiller-Zaoui model to a two phases composite (Eq. (6) eventually adapted to the infinite cylinder case)

\begin{tabular}{|c|c|c|c|c|c|c|}
\hline$\gamma^{\mathrm{p}}(\%)$ & $\tau$ & $f_{\mathrm{w}}$ & $x_{\mathrm{c}}($ exp.) & $x_{\mathrm{c}}(\mathrm{B}-\mathrm{Z})$ & $x_{\mathrm{c}}$ (Kröner) & Stress measurement \\
\hline \multicolumn{7}{|c|}{ Copper, well-oriented } \\
\hline 0.0015 & 17 & $0.6( \pm 15 \%)$ & $10( \pm 10 \%)$ & $0.65( \pm 37 \%)$ & 0.61 & Hysteresis loop \\
\hline 1 & 28 & $0.3( \pm 15 \%)$ & $11( \pm 10 \%)$ & $11.7( \pm 21 \%)$ & 96 & Hysteresis loop \\
\hline \multicolumn{7}{|c|}{ Austenitic steel 316L, well-oriented } \\
\hline 0.2 & 60 & $0.5( \pm 15 \%)$ & $16( \pm 10 \%)$ & $19( \pm 29 \%)$ & 54 & Hysteresis loop \\
\hline
\end{tabular}

Comparison experiment/model. Cyclic loading. Stress unit is MPa. Geometries: cylinders $(0.0015 \%$ and $0.2 \%)$ or equiaxed cells (0.28\% and 1\%). Experiment references: Mughrabi (1978), Laird et al. (1986), and Li and Laird (1994). Amplitudes of the backstresses are given.

geometry is considered: each (equiaxed) phase is supposed to be surrounded by the matrix one. The wall is still supposed to be elastic and the plasticity takes place only in the soft phase. Berveiller and Zaoui argumentation (1979) is now followed. The localization problem is reduced to a heterogeneity problem. For the sake of simplicity, the whole single crystal behaviour is modelled as an isotropic one and its secant behaviour is used. The application of the Berveiller and Zaoui model gives directly:

$$
\underline{\underline{x_{\mathrm{c}}}}=-2 \mu(1-\beta) \frac{1}{1+\frac{3}{2} \mu \frac{\varepsilon_{\mathrm{eq}}^{\mathrm{p}}}{\sigma_{\mathrm{eq}}}}\left(\frac{\varepsilon_{\mathrm{s}}^{\mathrm{p}}}{=}-\underline{\underline{\varepsilon^{\mathrm{p}}}}\right)
$$

Using the relationship between the soft phase plastic strain tensor and the macroscopic one (Eq. (2)), the modified backstress formula is obtained for a spherical cell:

$$
\underline{\underline{x_{\mathrm{c}}}}=-\frac{f_{\mathrm{w}}}{1-f_{\mathrm{w}}} \mu(1-\beta) \frac{1}{1+\frac{3}{2} \mu \frac{\varepsilon_{\mathrm{eq}}^{\mathrm{p}}}{\sigma_{\mathrm{eq}}}} \sum_{i=1}^{N} \gamma_{i}^{\mathrm{p}}\left(\underline{n_{i}} \underline{m_{i}^{T}}+\underline{m_{i}} \underline{n_{i}^{T}}\right)
$$

The accommodation factor (compare Eqs. (1) and (6)) is equal to:

$$
F_{\text {accom }}=\frac{1}{1+\frac{3}{2} \mu \frac{\varepsilon_{\mathrm{eq}}^{\mathrm{p}}}{\sigma_{\mathrm{eq}}}}
$$

It is similar to the classical Berveiller and Zaoui accommodation factor (Berveiller and Zaoui, 1979). It is quite equal to 1 for low plastic slips, which gives Eq. (4). But, if the 
$\varepsilon_{\text {eq }}^{p} / \sigma_{\text {eq }}$ coefficient is large enough, the accommodation factor is much lower than 1 . This accommodation factor is the quotient between the Berveiller and Zaoui prediction (Eq. (6)) and Kröner's prediction (Eq. (4)) (Tables 1 and 2). If only single slip is considered, a scalar close-form is obtained. This gives the shear backstress on the activated slip system:

$$
x_{\mathrm{c}}=-\frac{f_{\mathrm{w}}}{1-f_{\mathrm{w}}} \mu(1-\beta) \frac{1}{1+\frac{1}{2} \mu \frac{\gamma^{\mathrm{p}}}{\tau}} \gamma^{\mathrm{p}}
$$

with $\gamma^{\mathrm{p}}$ the plastic slip on the activated slip system.

Similar formulae can be given for other microstructure geometries, which can be modelled as homothetic concentric ellipsoids, introducing geometrical factors which depend on the ellipsoïd axes factors (Eshelby, 1957; Mura, 1987). For geometries more complicated than a sphere, Eqs. (2) and (3) can be modified. The $(1-\beta)$ coefficient and the accommodation factor can be modified (Mura, 1987). In addition, for multiple slip, a more complicated close-form is obtained because the backstress tensor can be no more proportional to the plastic strain tensor (case of infinite cylinders (Mura, 1987)).

For the validation of the model, two geometries have been used. They seem to be roughly representative of the two-phase dislocation microstructures corresponding to the tests and materials we refer to (Figs. 2 and 3). First, equiaxed cell microstructures have been considered (spherical cells, Fig. 2). For single slip (Eq. (8)) should be used whereas (Eqs. (6) and (7)) permits the computation of the backstress for multiple slip. Second, veins/channels microstructures are considered (hard phase kinds of cylinders embedded first in soft phase and second in matrix, Fig. 3). For the sake of simplicity, the cylinders are supposed to be purely elastic, infinite and to have a circular basis. They are supposed to be directly embedded in the macroscopic matrix and subjected to single slip. The slip normal and direction belong to the cylinder basis. This leads to relationship between the resolved shear stress and the primary plastic slip. For this second microstructure, the geometrical factor is equal to $1 /(4(1-v)$ ) (about 0.36 if $v=0.3$ ) instead of $(1-\beta)$ for the spherical cell (about 0.524 if $v=0.3$ ) (Pedersen, 1990; Mura, 1987). Following the idea given by Berveiller and Zaoui (1979), both macroscopic secant elastoplastic shear modulus and Poisson ratio are used instead of the elasticity coefficients in the Kröner thermoelastic solution. The backstress equation as well as its calculation are given in Appendix.

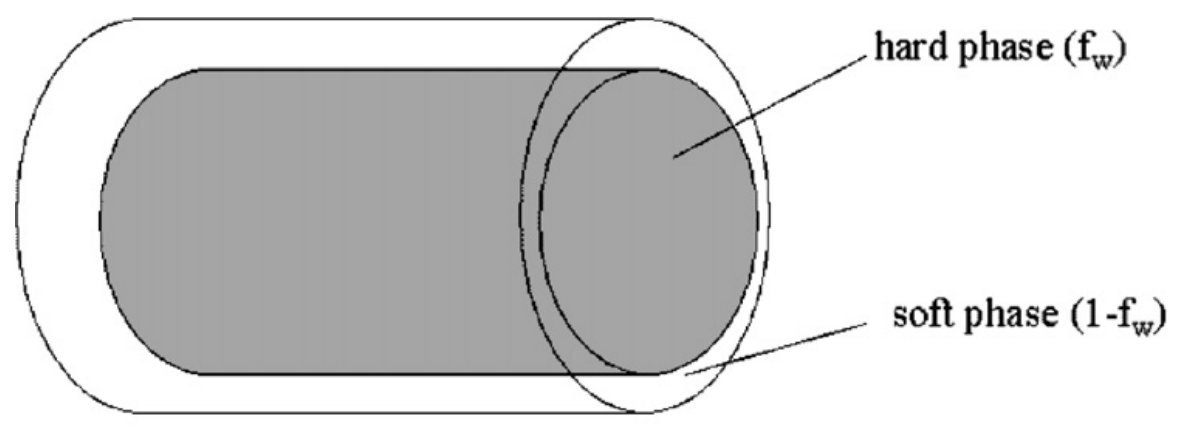

matrix

Fig. 3. Hard phase cylinder enrobed by soft phase (cyclic loading, small plastic strain). The real dislocation microstructure is made of less regular veins and channels. 
Some simplifications have been used in this model. The crystalline elasticity anisotropy has not been taken into account (cubic symmetry). The plastic anisotropy has neither been considered (for example single slip corresponds to an anisotropic plastic flow). And specific morphologies including a cell interior (inclusion), a concentric wall (shell) and a matrix can be modelled by some three-phase models (Hervé and Zaoui, 1990; Hervé et al., 1995) which consider separate inclusions (cell interiors) and a continuous phase (walls) (Figs. 2 and 3). But, it should be noted that if stresses and strains are considered as homogeneous in each phase, Eq. (4) (Kröner's approach) does not suppose any geometrical hypothesis such a cell embedded in a wall. If each phase is supposed to be directly embedded in the matrix one and if Kröner's localization rule is once more used, the same backstress value is obtained. Therefore, comparison between Eqs. (4) and (6) concerns rather the interaction hypothesis than the morphology hypothesis.

If more complicated hypotheses are considered (anisotropy, concentric walls), the computation are less straightforward and close-form formulae can no more be easily obtained. The choice of very simple hypotheses permit us to propose close-form formulae. This work is then more focused on close-form formulae and comparison with experimental measures.

\section{Validation for single crystals}

The validation is straightforward when considering tests and measurements on single crystals. For single crystals there are in fact no intergranular backstresses and the backstresses (inducing kinematic hardening) are reduced to the intragranular one. If there is no pile-up in the considered metals and alloys, the intragranular backstress is essentially due to the two-phase microstructure (walls and cell interiors/channels) and the plastic strain heterogeneities. Therefore the computed backstresses given in Eq. (4) or (6) (eventually adapted to the cylinder case) can be compared to the backstress experimental measures. There are many experimental means of measuring backstresses. There are at least three kinds of backstress measures: the mechanical one, the TEM one and the $X$-diffraction one.

First, the mechanical one uses the hysteresis $\gamma-\tau$ saturated loops (in the single slip case) (Cottrell, 1953; Kuhlmann-Wilsdorf and Laird, 1979; Feaugas, 1999). Considering the elastic part of the unloading curve, it is possible to measure the kinematic stress $x$ which is defined as the centre of the elasticity domain. It should be noticed that the kinematic stress is the backstress amplitude. The isotropic stress, $\tau_{\text {iso }}$, is the amplitude of the unloading elasticity domain which is described by the simple equation:

$$
|\tau-x|=\tau_{\text {iso }}
$$

The second experimental measure of backstress uses TEM observations (Essmann, 1965; Mughrabi, 1987). During or after a mechanical test, the specimen is irradiated by fast neutrons in order to pin up the dislocations. Measuring the dislocation radii and taking into account the edge/screw character of the dislocations, it is possible to evaluate the tension line using:

$$
\tau_{i}=\frac{T_{i}}{r_{i}}
$$

with $i=e, w$ depending on the edge/screw dislocation character, $T_{i}$ a coefficient depending on the elastic parameters and on the edge/screw dislocation character and $r_{i}$ is the 
dislocation radius. Finally, the backstress is determined using the dislocation equilibrium which gives $x_{\mathrm{s}}=\tau-\tau_{i}$ if the irradiation takes place at maximal load or $x_{\mathrm{s}}=-\tau_{i}$ if irradiation takes place after unloading. Backstresses of opposite signs are measured in the middle of the channels and near the walls. In the case of Persistent Slip Bands (PSBs) in copper well-oriented single crystals, the two experimental procedures agree quite well, giving a value of about $12 \mathrm{MPa}$ in the channels.

The third procedure uses $X$-diffraction (Mughrabi et al., 1986; Ungar et al., 1991). Copper single crystals oriented for multiple slip with $\langle 100\rangle$ axes have been deformed in tension

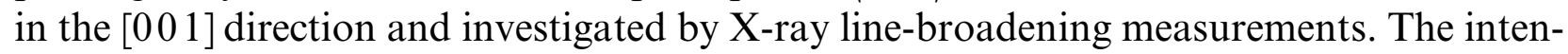
sity distributions of the broadened line profiles are asymmetric which is at least partially due to the two-phase nature of the deformed crystal. Two symmetric subprofiles corresponding to the walls and to the cell interiors can be exhibited. The displacements of the subprofiles with respect to the global profile have been evaluated by the authors in terms of elastic strains which permit them to obtain the long-range internal stresses in the walls and in the cell interiors, that is the backstresses.

For each backstress measure used for comparison with the predictions, the reference of the experimental study is indicated in the legends of Table 1 or Table 2 (Mughrabi et al., 1986; Ungar et al., 1991; Essmann, 1965; Fourie, 1968; Mughrabi, 1978; Laird et al., 1986; Li and Laird, 1994). The order of magnitude of the experimental scatter error is given too. For each measurement method, the scatter error is evaluated thanks to the scatter in the experimental data (Li and Laird, 1994; Mughrabi, 1987; Mughrabi et al., 1986).

In addition to the backstress measures, wall volume fractions, dislocation microstructure geometries (cells, walls/channels...) and average plastic slips are needed for applying Eq. (6) or (7). The wall volume fractions are usually obtained thanks to TEM observations and given in the referenced articles (Tables 1 and 2). In the case of the third procedure of backstress measurements (X-ray diffraction), the volume fractions are measured using the integral intensities of the subprofiles. These measurements seem to agree with the TEM ones (Mughrabi et al., 1986). The dislocation geometries are modelled taken into account TEM observations in different diffraction conditions in order to have a $3 \mathrm{D}$ view of the microstructures. The average plastic slips are given in the articles (Tables 1 and 2).

Concerning the computations, the case of single slip and multiple slip should be distinguished. The single slip case is straightforward using Eq. (8) with the plastic slips and shear stresses given in the articles. The multiple slip case needs more preliminary computations before using Eq. (6) which is more general than Eq. (8). Two cases have been considered. First, for the copper tensile test in the [0 011$]$ direction, symmetry and incompressibility reasons allow us to compute the plastic strain tensor which depends only on the plastic strain in the $[001]$ direction (or on the plastic slip which is the same for the eight activated slip systems (Anand et al., 1996)) for comparison with the experimental one. In this case, no crystallographic rotation occurs during deformation (Anand et al., 1996). Then, the backstress tensor is computed and the shear backstress is finally obtained after projection of the backstress tensor on one of the activated slip system. Second, concerning the tensile test on a well-oriented crystal, the plastic slips on the activated slip systems have been computed by Anand et al. (1996) which permits us to evaluate the plastic strain tensor components (at the beginning of the test, only the well-oriented slip system is activated, but after large plastic deformation, a second one is activated because of the large rotation of the slip systems). And the shear backstress obtained on the well-oriented slip system is finally deduced 
as before. It should be noticed that Eq. (1) is not strictly valid in this case because of the crystallographic rotation occurring during tensile deformation (an incremental formulation should be used).

The measured and computed backstresses are given in Tables 1 and 2. Computations are made thanks to Eq. (6) or (8). The computed accommodation factor, $F_{\text {accom}}$, is given too. Table 1 is dedicated to multiple slip and tensile loadings. Table 2 focuses on single slip and cyclic loadings. Several metals (copper, 316L stainless steel), orientations (single slip, multiple slip) and test conditions (tensile/cyclic loadings, small/large plastic strains) have been considered. Rough estimations of the experimental catter errors on the measured backstresses and hard phase volume fractions are given in Tables 1 and 2. These estimations are based on the scatter in the data we could find in the corresponding articles. The error ranges on the computed backstresses induced by the experimental scatter errors of the dislocation wall volume fractions have been computed and are given in Tables 1 and 2. The predictions are reasonable except in the case of very small plastic strains (of order of magnitude $10^{-5}$ ).

Finally, the whole evolution of the computed backstress along the tensile deformation of a copper [ 001 1] single crystal is plotted in Fig. 4. Only measured values of the hard phase volume given by Mughrabi et al. are used in our computations (either using TEM observations or X-ray diffraction, Mughrabi et al., 1986). The predicted evolution can be compared with the evolution of the backtress measured by Ungar et al. by X-ray diffraction (Ungar et al., 1991). Taking into account the scatter errors, the computations agree quite well with the experiments. For the smallest macroscopic shear stress value, it should be noticed that the scatter error on the measured backstress seems to be rather high following Fig. 8 of Mughrabi et al., 1986. The relative scatter error seems smaller at higher shear stress. But, for plotting the experimental error bars, a constant relative scatter error of $15 \%$ was used even if the relative error could be larger at the smallest stress amplitude.

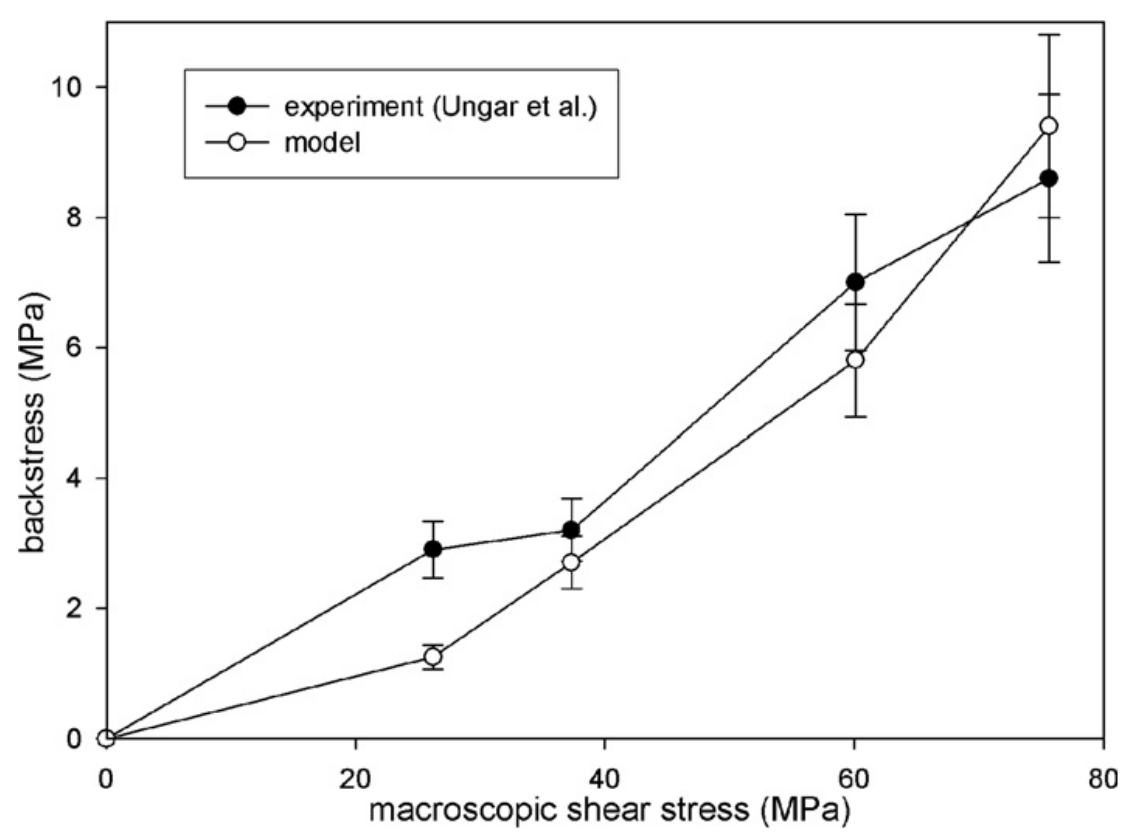

Fig. 4. Comparison between computed and measured backstresses. Tensile deformation of a [001] copper single crystal. Experimental measures: Ungar et al. (1991), for the backstresses and Mughrabi et al. (1986), for the hard phase volume fractions. Model of spherical cells (Fig. 2). Amplitudes of the backstresses are given. 


\section{Discussion}

Comparison between Kröner's predictions and experimental measures confirms the inadequacy of the classical inclusion models of intragranular backstresses (Tables 1 and 2). In fact, they suppose thermoelastic interactions between the phases and the average medium, that is why they predict too high backstresses. Even in the case of plastic strain with an order of magnitude of $10^{-3}$, Kröner's analysis gives too high backstresses (two or three times the experimental ones) (Table 2). But, in this case, the Berveiller and Zaoui approach gives backstresses which are rather close to the experimental ones (computed values using B-Z, Table 2, Fig. 4). For large plastic strains, Kröner's predictions are two orders of magnitude higher than the measured values whereas the Berveiller and Zaoui predictions are much more reasonable. The ratio between the two models is in that case larger than 100. Generally, the agreement between the close-form Berveiller and Zaoui formulae and the measured values is rather encouraging.

But, in the case of very small plastic strains (of order of magnitude $10^{-5}$ ), the model underestimates largely the backstress. Computation using Eq. (8) gives in fact a backstress with an order of magnitude smaller than the experimental one. In addition, the model supposes that walls are dense enough for being considered as elastic. In fact, there seems to be only a loose heterogeneous dislocation microstructure (Laird et al., 1986; Obrtlik et al., 1993), which means that the computation should predict an overestimation of the backstress induced by the dislocation microstructure. Therefore, following Table 2, there is a real discrepancy between the prediction and the measured value. In fact, there can be other types of backstress sources such as dislocation pile-ups. For small applied stress, there is less cross-slip than for large stresses. That is why pile-ups could be more efficient for small applied stresses and could induce backstresses which are not taken into account in Eq. (6) or (8).

The intragranular kinematic hardening model corresponding to Eq. (2) can be easily used when applying an analytical/numerical polycrystalline homogenization model. These homogenization models allow us to compute the macroscopic behaviour depending on the texture and on the intragranular constitutive laws. For taking into account the interactions between grains, an intergranular backstress computation model has to be used too (see for example (Holden et al., 1998)). Thanks to the intragranular kinematic hardening model, the intragranular backstress tensor can be computed in each grain (that means for each crystallographic orientation). It depends on the stresses and plastic strains values in the considered grain. In fact, the mechanical fields and hardening variables at the grain scale are known only after the resolution of a global equation system concerning all orientations. It should be noticed that the proposed intragranular kinematic hardening model needs no adjustable parameter contrary to other models (Feaugas and Gaudin, 2001; Harder, 1999). Generally, in the framework of elastoplasticity, polycrystalline homogenization models include two parts: grain-polycrystal homogenization rules and crystalline constitutive equations (Cailletaud, 1992). Then, they need numerous adjustable parameters. Therefore it is often uneasy to compare polycrystalline predictions with experimental results. If a disagreement is for example observed, it is not clear how to improve predictions. Should it concern the grain-polycrystal localization rule? The crystalline isotropic hardening model? Or the crystalline kinematic one? If each specific part of the global model is validated independently, it could permit an easier research of the global model possible improvement. As an example of the backstress model interest, the predictions 
of the intragranular and intergranular backstresses given by Feaugas (1999) is now considered. The authors predict a vanishing of the intergranular backstresses with tensile plastic strain increasing. These results are in disagreement with the evolution measured by Holden et al. (1998) in similar conditions. For comparison, intragranular and intergranular backstress evolutions are computed using Eq. (6) and wall volume fractions measured by Feaugas (1999) thanks to TEM observations. A stabilization of both is predicted which agrees much better with the experimental results of Holden et al. (1998). A crystalline kinematic hardening model and its parameters are needed for performing Finite Element crystalline computations too (Barbe et al., 2001; Clayton and McDowell, 2003; Erieau and Rey, 2004; Bertolino et al., 2005). Recently, some enhanced models were proposed for simulating forming processes (Tugcu et al., 2004; Habraken and Duchêne, 2004; Raabe and Roters, 2004). Both finite element computations at the structure scale and grain-volume element transition laws are used. But these models did not take into account any physically based intragranular kinematic hardening law even if it is well-known that large plastic strains induce cell formation and long-range internal stresses. In addition, it has been noticed that the intragranular kinematic hardening mechanisms could affect significantly the macroscopic behaviour during change of loading path (Langlois and Berveiller, 2003). Then, the proposed model (Eqs. (6) and (8)) could help to carry out simulations using an additional microstructure mechanism.

But, this model needs knowledge about the deformation induced microstructures (wall volume fraction, $f_{\mathrm{w}}$, and microstructure morphology). As mentioned before, the $f_{\mathrm{w}}$ volume fraction is an influent parameter of the intragranular backstress model. The evaluation of the $f_{\mathrm{w}}$ volume fraction has to be precise because the backstress is proportional to the $f_{\mathrm{w}} /$ $\left(1-f_{\mathrm{w}}\right.$ ) factor (Eq. (6)) of which variation with $f_{\mathrm{w}}$ is large. If a $f_{\mathrm{w}}$ variation of $\pm 10 \%$ is supposed, a backstress variation of $\pm 14 \%( \pm 21 \%)$ is obtained for $f_{\mathrm{w}}=0.3\left(f_{\mathrm{w}}=0.5\right)$. For the validation, experimental measures of $f_{\mathrm{w}}$ have been used, originating from either TEM observations or the X-rays diffraction measures. These volume fraction measures obtained by these two techniques seem to agree following Mughrabi et al. (1986). In order to propose a complete backstress prediction, the use of a deformation induced dislocation microstructure model is needed, allowing the prediction of the hard phase morphology and volume fraction. Then, the backstress model could be applied. The dislocation microstructure characteristics depend in fact on both the material and loading. A large number of dislocation microstructure prediction models have been proposed. At least three kinds can be distinguished. First, they can use an energy minimization principle. Following this principle, the observed two-phase dislocation microstructure is the less energetic microstructure (Kuhlmann-Wilsdorf and van der Merwe, 1982; Kuhlmann-Wilsdorf, 2001). Second, they can be founded on bifurcation analysis. For example, if two equilibrium solutions are possible, one can be stable (two-phase microstructure) and the other one can be unstable (homogeneous microstructure) and therefore can not be observed (Kratochvil, 1990). Third, continuous or discrete dislocation dynamics models try to predict the formation of dislocation microstructures (Differt and Essmann, 1993; Pantleon, 1998; Robertson et al., 2001). These models generally take into account dislocation interaction, annihilation and dipole formation. The recent modelling attempt of Zhou et al. (2006), should be mentioned. These authors try to predict the volume fraction of PSBs in nickel single crystals using crystalline finite element analysis.

The possibility of plastic glide inside the dense walls is now discussed. Indeed, authors do not agree completely about wall plasticity. The walls are considered to be plastically 
deformed by some authors (Mughrabi, 1987; Feaugas and Gaudin, 2001), but they are supposed to be purely elastic by others (Pedersen, 1990; Langlois and Berveiller, 2003). Because of their high dislocation densities, the walls are less deformable than the soft phase and their critical shear stress is higher. Plastic incompatibilities induce long-range stresses inside both the soft phase (backstress $x_{\mathrm{c}}$ ) and the hard phase, $x_{\mathrm{w}}$ (Mughrabi, 1983). The wall long-range stress ("forward stress") magnitude is generally high because of their small volume fraction (the single crystal average long-range stress is equal to zero (Mura, 1987)). Cell backstress and wall forward stress have opposite signs. Therefore, contrary to the soft phase backstress, the hard phase forward stress tends to aid plastic glide at the end of loading/unloading. Finally, is the wall critical stress reached during loading/ unloading? This would induce dislocation glide inside the hard phase which should be taken into account in the modelling. Hard phase effective stresses can be computed and compared with wall critical shear stress for guessing if walls could be plastically deformed or not. The soft phase backstress predictions (Tables 1 and 2) allow us to compute the hard phase "forward stress" values (Tables 3 and 4) because the average long-range internal stress should be equal to zero. Inside the walls, at the end of loading/unloading, the effective stress is the sum of the applied stress and the local forward stress because they have the same sign. Then, well-known critical shear stress models are used, corresponding to the microstructures observed in some of the experiments used for the model validation (Tables 1 and 2). Two local hardening mechanisms are considered. First, well-oriented single crystals tested at small cyclic strain/stress are studied. Two microstructures are usually observed, depending on the applied strain: either veins/channels (Fig. 3) only or persistent slip bands (PSBs) and veins/channels (Lukas et al., 1968). Following TEM observations, the walls are made of edge dipoles for both. Following the Essmann and Differt dipole hardening model (1996), the critical shear stress can be calculated:

$$
\tau_{\text {crit, } \mathrm{w}}=\beta D\left\langle y_{\mathrm{d}}\right\rangle \frac{\rho_{\mathrm{w}}}{2}
$$

with $\beta$ a constant depending on the dipoles-mobile dislocation interaction kind (on average $\beta \approx 5), D=\mu b / 2 \pi(1-v)$ and $\left\langle y_{\mathrm{d}}\right\rangle$ the average dipole height. This is the minimal stress for dislocation gliding through the wall dipoles. The average dipole height is given by:

Table 3

Forward stress, effective stress and critical shear stress inside the walls at maximal load

\begin{tabular}{lllll}
\hline Microstructure & $x_{\mathrm{w}}$ & $\tau+x_{\mathrm{w}}($ effective $)$ & $\tau_{\text {crit,w }}($ dipoles $)$ & $\rho_{\mathrm{w}}\left(\times 10^{15} \mathrm{~m}^{-2}\right)$ \\
\hline Veins/channels & 12 & 29 & 72 & 1.5 \\
PSBs & 70 & 100 & $125-250$ & $3-6$ \\
\hline
\end{tabular}

Copper, low strain cycling. Dipole hardening model (single slip) (Essmann and Differt, 1996). Stress unit is MPa.

Table 4

Forward stress, effective stress and critical shear stress inside the walls at maximal load

\begin{tabular}{lllll}
\hline Microstructure & $x_{\mathrm{w}}$ & $\tau+x_{\mathrm{w}}($ effective $)$ & $\tau_{\text {crit,w }}$ (forest) & $\rho_{\mathrm{w}}\left(\times 10^{15} \mathrm{~m}^{-2}\right)$ \\
\hline Cells (fatigue) & 60 & 90 & $130-245$ & $1-6$ \\
Cells (monotonic) & 30 & 105 & 109 & 0.79 \\
\hline
\end{tabular}

Copper, high strain cycling and tensile loading with high strain. Equiaxed cells. Forest hardening model (multiple slip). Stress unit is $\mathrm{MPa}$. 


$$
\left\langle y_{\mathrm{d}}\right\rangle=\frac{y_{\text {max }} y_{\mathrm{e}}}{y_{\text {max }}-y_{\mathrm{e}}} \ln \left(\frac{y_{\text {max }}}{y_{\mathrm{e}}}\right)
$$

with $y_{\mathrm{e}}$ the edge annihilation distance $\left(y_{\mathrm{e}}=1.6 \mathrm{~nm}\right.$ for copper at room temperature) and $y_{\max }$ the maximal stable dipole height. Considering a local shear stress equal to $\tau_{\text {loc }}$, dipole stability is assured if the dipole height is smaller than the maximal one which can be computed too (Essmann and Differt, 1996):

$$
y_{\max }=\frac{D}{4 \tau_{\text {loc }}}
$$

The computed critical stresses are given in Table 3. The wall dislocation densities are given in the corresponding articles (Table 3). Isotropic elasticity coefficients have been used. For the veins/channels microstructure, the computed critical shear stress is larger than the effective stresses and it seems not very easy to activate dislocation glide inside the walls. But, for PSB walls, it is impossible to conclude because the effective stress and the critical shear stress values could be rather close or far depending on the dislocation density measure for which there is always non-negligible experimental scatter.

Second, high strain cyclic loadings or tensile loadings are considered. They induce equiaxed cell formation in the studied cases (Tables 2 and 4) (Fig. 2). As multiple slip is activated in such cases, a forest hardening model is used for computing the critical shear stress inside the walls:

$$
\tau_{\mathrm{c}, \mathrm{w}}=\alpha \mu b \sqrt{\rho_{\mathrm{w}}}
$$

A classical forest hardening coefficient of $\alpha=0.3$ has been used. The wall dislocation densities are given in the corresponding articles (Table 4). Isotropic elasticity coefficients have been used. The computed critical shear stresses are larger than the effective stresses (Table 4). But, the differences are rather small and for the tensile loading the effective stress is nearly equal to the flow stress. Indeed the hardening parameter values are not known precisely, that is why general conclusions can not be drawn from these comparisons except for the vein/channel microstructure. Finally, it should be noticed that the backstresses computed using either Eq. (6) or (8) become proportional to the total stress for large enough plastic strains (and if the dislocation microstructures do not change a lot with the strain increasing). If the effective stress saturates too, then the total stress remains constant and the behaviour is a perfectly plastic one. Mughrabi claims that the perfect plasticity behaviour which is observed for copper single crystals at the end of high strain experimental cycle loading indicates that the walls deform plastically at high strain (Mughrabi, 1988). In fact, our model could predict such perfect plasticity even if walls deform elastically. Of course, the saturated stress level depends on the behaviour of the walls (elasticity or elastoplasticity).

For the sake of simplicity, both elastic and plastic anisotropies of single crystals are neglected when using the Berveiller and Zaoui localization rule. In fact, both the single crystal elasticity and plasticity are supposed to be isotropic. For copper and austenite, the cubic elasticity anisotropy ratio is about 3.3 which means that the elasticity anisotropy is high. Crystalline plasticity is neither isotropic because of the finite number of slip systems. Work is in progress for taking into account these anisotropy sources. But, it should be noticed that it would no more permit us to give close-form formulae and to propose straightforward computations of the backstresses. In fact, the computation of the Eshelby 
localization coefficients would be needed. And for anisotropic elasticity, their computation requires generally the use of numerical procedures (Mura, 1987).

When using the Berveiller and Zaoui localization rule, the enrobed character of the soft (or hard) phase has been neglected too (Figs. 2 and 3). But, it could influence the soft phase backstresses and consequently the intragranular kinematic hardening. Lemoine et al. model takes this particular morphology into account (Lemoine et al., 1994). But, the deduced soft phase backstress is the same as for a simpler morphology with soft phase spherical inclusions directly embedded in the macroscopic medium (like for the two-phase model described in this paper). In fact, it takes into account only the wall volume fraction and the soft phase ellipsoidal geometry. And their model is thermoelastic, it does not reproduce any accommodation effect and therefore gives much larger backstress values as soon as the plastic strain is higher than a few $10^{-4}$ (Table 2). Following the Hervé and Zaoui approach (three phase inclusion-matrix model), work is in progress for taken into account both the real morphologies of dislocation microstructures and the plastic accommodation (Hervé and Zaoui, 1990). Comparisons with possible predictions of such enhanced models can already be discussed. Microstructures considered in this article are of two kinds. First, dislocation equiaxed cells can be modelled as soft phase inclusion/hard shell/matrix geometries (Fig. 2). Second, veins/channels microstructures can be modelled as hard phase cylindrical inclusion/soft phase shell/matrix geometries (Fig. 3). Following the published results concerning the three phase inclusion-matrix model and referring to the two-phase Berveiller and Zaoui model predictions (this article ones), higher stresses should be predicted for the first kind of microstructure, and smaller for the second kind (see (Hervé and Zaoui, 1990) concerning elastoplasticity and (Hervé et al., 1995) concerning viscoplasticity). Therefore, for the microstructures described in Table 1 and for the second one of Table 2 (dislocation cells), higher backstresses should be predicted. But, if differences between three-phase and two-phase models were high, predictions obtained thanks to the three-phase model would not agree with experimental measures. For the two remaining microstructures of Table 2 (vein/channel microstructures), smaller backstresses would be predicted, which would not considerably improve the prediction-experiment comparison. In fact, considering the parameters of the studied dislocation microstructures and the comparisons between both models published in the literature, small differences between both could be predicted. Following (Hervé et al., 1995), the smaller is the contrast between the hard and soft phase (in terms of reference stress ratios), the smaller is the relative difference between the two-phase and the three-phase model predictions (in the viscoplasticity framework). Let us first consider soft inclusion-hard shell geometries. Following Tables 3 and 4, the critical shear stress in the walls of copper single crystals is in the 75-250 $\mathrm{MPa}$ range depending on the dislocation microstructure. The critical shear stress in the soft phase is probably close to $15 \mathrm{MPa}$ (critical shear stress measured during the tensile loading of a copper single crystal (Anand et al., 1996), amplitude of the hysteresis loops (Mughrabi, 1978)). It means that the ratio between the critical shear stresses of the hard and soft phases is in the 5-17 range. Following the computations reported in (Hervé et al., 1995), for a ratio equal to 10, a viscoplasticity exponent equal to 0.5 in both phases and for wall volume fractions in the $0.3-0.5$ range (see Tables 1 and 2), the difference between the macroscopic reference stresses obtained thanks to a two-phase or a three-phase model is relatively small. The same holds for the case of the cylindrical geometries mentioned in Table 2 (hard inclusion, soft shell). The stress state in the walls should be particularly studied in order to better evaluate if 
plastic glide is acting inside them (see Section 4 above about this subject). Work is in progress for comparing predictions of three-phase and two-phase models. But, it should be noticed that probably no analytical expression of the backstress could be available using the three-phase model which requires numerical computations (Hervé and Zaoui, 1990).

\section{Conclusions}

A large number of experiments have shown that deformation induced dislocation microstructures (hard and soft phases appearing for example in FCC metals and alloys) induce backstresses at the single crystal scale. In this article, an application of the Berveiller and Zaoui localization scheme is proposed. It is based on the use of the single crystal secant modulus and on a deduced accommodation factor. It allows us to take into account non-negligible plastic strains. Eshelby's inclusion problem solutions are used for two kinds of geometry: either spherical soft phase inclusions or hard phase infinite cylinders. The model is specifically validated at the single crystal scale because in this case, only intragranular backstresses are involved in the kinematic hardening. For the validation, a large number of backstress experimental measures on single crystals after tensile or cyclic loadings are used. The backstress model needs no adjustable parameter and only experimental data are used for the computation. Despite the Berveiller and Zaoui localization rule does not take into account crystalline elasticity and plasticity anisotropies, the agreement between predictions and measures is rather encouraging. Its predictions are much more reasonable than the ones obtained using classical inclusion models of intragranular backstresses. Thanks to the rudimentary hypotheses of the model, analytical formulae can be proposed and the computations are straightforward. This intragranular kinematic hardening model could replace more phenomenological models and avoids the use of any adjustable parameter. Because of its physical basis, it could help to improve the predictions of polycrystalline homogenization codes or crystalline finite element codes which need the knowledge of intragranular constitutive laws.

\section{Acknowledgements}

A. Pineau, R. Brenner and X. Feaugas are acknowledged for stimulating discussions and I. Monnet for giving Fig. 1 (TEM observation).

\section{Appendix A. Calculation of the backstress equation for veins and channels microstructures subjected to single slip}

Veins/channels microstructures are considered (hard phase kinds of cylinders embedded first in soft phase and second in matrix, Fig. 3). For the sake of simplicity, the cylinders are supposed to be purely elastic, infinite and to have a circular basis. They are supposed to be directly embedded in the macroscopic matrix. Only single slip is considered. The slip normal and direction belong to the cylinder basis. This leads to resolved shear stress versus primary plastic slip relationships. The macroscopic plastic slip is denoted as $\gamma^{\mathrm{p}}$. For the cylinder inclusion, the geometrical factor is equal to $1 /(4(1-v))$ (about 0.36 if $v=0.3$ ) instead of $(1-\beta)$ for the spherical cell (about 0.524 if $v=0.3$ ) (Pedersen, 1990; Mura, 1987). A computation similar to the one described by Berveiller and Zaoui is carried out for calculating the evaluating accommodation induced by the 
use of secant instead of elasticity coefficients (Berveiller and Zaoui, 1979). The Eshelby stress solution is computed using the secant elastoplastic shear modulus and Poisson ratio, $\mu_{\text {secant }}$ and $v_{\text {secant }}$, instead of the isotropic elasticity moduli, $\mu$ and $v$. Following Kröner's idea, the inclusion free strain is replaced by the difference between the inclusion and matrix plastic strains (Kröner, 1961), which reduces to the opposite of the macroscopic plastic strain, $-\gamma^{\mathrm{p}} / 2$, because the cylinders are purely elastic. Therefore, the residual stress in the hard phase, $x_{\mathrm{w}}$, can be computed using:

$$
x_{\mathrm{w}}=\mu_{\text {sec ant }} \frac{1}{4} \frac{1}{\left(1-v_{\text {sec ant }}\right)} \gamma^{\mathrm{p}}
$$

The residual resolved shear stress in the soft phase, i.e. the backstress, $x_{\mathrm{c}}$, is deduced using average stress balance equation:

$$
f_{\mathrm{w}} x_{\mathrm{w}}+\left(1-f_{\mathrm{w}}\right) x_{\mathrm{c}}=0
$$

Finally, the backstress can be computed as follows:

$$
x_{\mathrm{c}}=-\frac{f_{\mathrm{w}}}{1-f_{\mathrm{w}}} \mu_{\text {sec ant }} \frac{1}{4} \frac{1}{\left(1-v_{\text {sec ant }}\right)} \gamma^{\mathrm{p}}
$$

It depends only on the elasticity coefficients and on the macroscopic plastic slip and shear stress, $\gamma^{\mathrm{p}}$ and $\tau$, because the secant elastoplastic shear modulus and Poisson ratio depend only on the macroscopic stress and plastic slip (Berveiller and Zaoui, 1979):

$$
\begin{aligned}
\mu_{\text {sec ant }} & =\frac{\tau}{\frac{\tau}{\mu}+\gamma^{p}}=\mu \frac{1}{1+\mu \frac{\gamma^{p}}{\tau}} \\
v_{\text {sec ant }} & =\frac{v+\mu(1+v) \frac{\gamma^{p}}{3 \tau}}{1+2 \mu(1+v) \frac{\gamma^{p}}{3 \tau}}
\end{aligned}
$$

Using the Young's modulus, $E=2(1+v) \mu$ and von Misès equivalent plastic strain and stress, $\varepsilon_{\mathrm{eq}}^{p}=\gamma^{p} / 3^{1 / 2}$ and $\sigma_{\mathrm{eq}}=3^{1 / 2} \tau$ (single slip). Elasticity is supposed to be isotropic. Plasticity transformation is isovolumetric and isotropic.

\section{References}

Anand, L., Balasubramanian, S., Kothari, K., 1996. Constitutive modeling of polycrystalline metals at large strains. In: Teodosiu, C. (Ed.), CISM Courses and Lectures No. 376: Large Plastic Deformation of Crystalline Aggregates, p. 109.

Barbe, F., Forest, S., Cailletaud, G., 2001. Intergranular and intragranular behavior of polycrystalline aggregates. Part 1. FE model. Int. J. Plast. 17, 513-536.

Berveiller, M., Zaoui, A., 1979. An extension of the self-consistent scheme to plastically-flowing polycrystals. J. Mech. Phys. Solids 26, 325-344.

Bertolino, G., Doquet, V., Sauzay, M., 2005. Modelling of the scatter in short fatigue cracks growth kinetics in relation with the polycrystalline microstructure. Int. J. Fat. 27, 471-480.

Beyerlein, I.J., Tomé, C.N., 2007. Modeling transients in the mechanical response of copper due to strain path changes. Int. J. Plast. 23 (4), 640-664.

Cailletaud, G., 1992. A micromechanical approach to inelastic behaviour of metals. Int. J. Plast. 8, 55-73.

Clayton, J.D., McDowell, D.L., 2003. A multiscale multiplicative decomposition for elastoplasticity of polycrystals. Int. J. Plast. 19, 1401-1444.

Cottrell, A.H., 1953. Dislocations and Plastic Flow in Crystals. Oxford University Press, London, p. 111.

Delannay, L., Jacques, P.J., Kalidindi, S.R., 2006. Finite element modeling of crystal plasticity shaped as truncated octahedrons. Int. J. Plast. 22, 1879-1898. 
Déprés, Ch. 2004. Modélisation physique des stades précurseurs de l'endommagement en fatigue dans l'acier inoxydable austénitique 316L. PhD, Institut National Polytechnique de Grenoble, Grenoble, France.

Differt, K., Essmann, U., 1993. Dynamical model of the wall structure in persistent slip bands of fatigued metals I. Dynamical model of edge dislocation walls. Mater. Sci. Eng. A 164, 295-299.

Erieau, Ph., Rey, C., 2004. Modeling of deformation and rotation bands and of deformation induced grain boundaries in IF steel aggregate during large plane strain compression. Int. J. Plast. 20, 1763-1788.

Eshelby, J.D., 1957. The determination of the elastic field of an ellipsoidal inclusion. Proc. Roy. Soc. A 252, 561569.

Essmann, U., 1965. Electron microscopy investigation of dislocation arrangements in copper single crystals, Part I and Part II. Phys. Status Solidi 12 (2), 707-722 (Part I), 723-747 (Part II).

Essmann, U., Differt, K., 1996. Dynamic model of the wall structure in persistent slip bands of fatigued metals II. The wall spacing and the temperature dependence of the yield stress in saturation. Mater. Sci. Eng. A 208, 5668.

Feaugas, X., 1999. Contribution à la compréhension des mécanismes de déformation plastique et d'endommagement des matériaux. In: Habilitation à Diriger des Recherches. Université de Compiègne, Compiègne, France.

Feaugas, X., 1999. On the origin of the tensile flow stress in the stainless steel AISI 316L at 300K: backstress and effective stress. Acta Mater. 47, 3617-3632.

Feaugas, X., Gaudin, C., 2001. Different levels of plastic strain incompatibility during cyclic loading: in terms of dislocation density and distribution. Mater. Sci. Eng., 382-385.

Fourie, J.T., 1968. The flow stress gradient between the surface and the centre of deformed single crystals. Phil. Mag. 17, 735-756.

Gerland, M., Mendez, J., Violan, P., Ait Saadi, 1989. Evolution of dislocation structures and cyclic behaviour of a 316L-type austenitic steel cycled in vacuo at room temperature. Mater. Sci. Eng. A 118, 83-95.

Guan, Y., Pourboghrat, F., Barlat, F., 2006. Finite element modeling of tube hydroforming of polycrystalline aluminium alloy extrusions. Int. J. Plast. 22, 2366-2393.

Habraken, A.-M., Duchêne, L., 2004. Anisotropic elastoplastic finite element analysis using a stress-strain interpolation method based on a polycrystalline model. Int. J. Plast. 20, 1525-1560.

Hansen, N., Huang, X., 1998. Microstructure and flow stress of polycrystals and single crystals. Acta Mater. 46, $1827-1836$.

Harder, J., 1999. A crystallographic model for the study of local deformation processes in polycrystals. Int. J. Plast. 15, 605-624.

Hervé, E., Zaoui, A., 1990. Modelling the effective behavior of non-linear matrix-inclusion composites. Eur. J. Mech., A/Solids 9 (6), 505-515.

Hervé, E., Dendievel, R., Bonnet, G., 1995. Steady-state power-law creep in "inclusion-matrix" composite materials. Acta Metall. Mater. 43, 4027-4034.

Holden, T.M., Holt, R.A., Clarke, A.P., 1998. Intergranular strains in Inconel-600 and the impact on interpreting stress fields in bent steam-generator tubing. Mater. Sci. Eng. A 246, 180-198.

Khan, S.M.A., Zbib, H.M., Hugues, D.A., 2004. Modeling planar dislocation boundaries using multi-scale dislocation dynamics plasticity. Int. J. Plast. 20, 1059-1092.

Kröner, E., 1961. On the plastic deformation of polycrystals. Acta Metall. 9, 155-161.

Kratochvil, J., 1990. Derivation of Mughrabi's cellular structure model from synergetics of dislocations. Scripta Met. Mater. 24, 891-894.

Kuhlmann-Wilsdorf, D., Laird, C., 1979. Dislocation behavior in fatigue II. Friction stress and back stress as inferred from an analysis of hysteresis loops. Mater. Sci. Eng. 37, 111-120.

Kuhlmann-Wilsdorf, D., van der Merwe, J.H., 1982. Theory of dislocation cell sizes in deformed metals. Mater. Sci. Eng. 55, 79-83.

Kuhlmann-Wilsdorf, D., 2001. Q: Dislocations structures - how far from equilibrium? A: Very close indeed. Mater. Sci. Eng. A 315, 211-216.

Laird, C., Charsley, Ph., Mughrabi, H., 1986. Low energy dislocation structures produced by cyclic deformation. Mater. Sci. Eng. 81, 433-450.

Langlois, L., Berveiller, M., 2003. Overall softening and anisotropy related with the formation and evolution of dislocation cell structures. Int. J. Plast. 19, 599-624.

Lemaitre, J., Chaboche, J.-L., 1987. Mechanics of Solid Materials. Springer-Verlag.

Lemoine, X., Muller, D., Berveiller, M., 1994. Texture of microstructure in BCC metals for various loading paths. Mater. Sci. Forum 157-162, 1821-1826. 
Li, Y., Laird, C., 1994. Cyclic response and dislocation structures of AISI 316L stainless steel. Part 1. Single crystals fatigued at intermediate strain amplitude. Mater. Sci. Eng. A 186, 65-86.

Lukas, P., Knesnil, M., Krejci, J., 1968. Dislocations and persistent slip bands in copper single crystals fatigued at low-stress amplitude. Phys. Stat. Sol. 27, 545-558.

Mughrabi, H., 1978. The cyclic hardening and saturation behaviour of copper single crystals. Mater. Sci. Eng. 33, 207-223.

Mughrabi, H., 1983. Dislocation wall and cell structures and long-range internal stresses in deformed metal crystals. Acta Metall. 31 (9), 1367-1379.

Mughrabi, H., Ungar, T., Kienle, W., Wilkens, M., 1986. Long-range internal stresses and asymmetric X-ray linebroadening in tensile-deformed [001]-oriented copper single crystals. Phil. Mag. A 53 (6), 793-813.

Mughrabi, H., 1987. The long-range internal stress field in the dislocation wall structure of persistent slip bands. Phys. Stat. Sol. (a) 104, 107-120.

Mughrabi, H., 1988. Dislocation clustering and long-range internal stresses in monotonically and cyclically deformed metal crystals. Rev. Phys. Appl. 23, 367-379.

Mura, T., 1987. Micromechanics of defects in solids. Martinus Nijhoff Publishers, Dordrecht.

Nakamachi, E., Tam, N.N., Morimoto, H., 2007. Multi-scale finite element analysis of sheet metals by using SEM-EBSD measured crystallographic RVE models. Int. J. Plast. 23 (3), 450-489.

Obrtlik, K., Polak, J., Komurka, J., 1993. Dislocation structures in polycrystalline copper cycled at low strain amplitudes. Scripta Met. Mater. 28, 495-499.

Pantleon, W., 1998. On the statistical origin of disorientation in dislocation microstructures. Acta Mater. 46 (2), 451-456.

Pedersen, O.B., 1990. Mechanism maps for cyclic plasticity and fatigue of single phase materials. Acta Metall. Mater. 38 (7), 1221-1239.

Polak, J., 1991. Cyclic Plasticity and Low-Cycle Fatigue Life of Metals. Elsevier Science Publishers, Amsterdam.

Polak, J., Obrtlik, K., Hajek, M., Vasek, A., 1992. Cyclic stress-strain response of polycrystalline copper in a wide range of plastic strain amplitudes. Mater. Sci. Eng. A 151, 19-27.

Raabe, D., Roters, F., 2004. Using texture components in crystal plasticity finite element simulations. Int. J. Plast. 20, 339-361.

Robertson, C., Fivel, M.C., Fissolo, A., 2001. Dislocation substructure in 316L stainless steel under thermal fatigue up to $650 \mathrm{~K}$. Mater. Sci. Eng. A 315, 47-58.

Sauzay, M. 2000. Effets de surface et d'anisotropie en fatigue multiaxiale. Thèse de l'Université Paris 6, France, p. 54.

Tabata, T., Yamanaka, S., Fujita, H., 1977. In situ deformation of the [1 11] aluminium single crystals observed by high voltage electron microscopy. Acta Metall. 26, 405-411.

Tabata, T., Fujita, H., Hiraoka, M.-A., Miyake, S., 1982. The relationship between flow stress and dislocation behaviour in [1 11 ] aluminium single crystals. Phil. Mag. A 46, 801-816.

Tugcu, P., Neale, K.W., Wu, P.D., Inal, K., 2004. Crystal plasticity simulation of the hydrostatic bulge test. Int. J. Plast. 20, 1603-1653.

Ungar, T., Mughrabi, H., Wilkens, M., Hilscher, A., 1991. Long-range internal stresses and asymmetric X-ray line broadening in tensile-deformed [0 01 1]-oriented copper single crystals: the correction of an erratum. Phil. Mag. A 54 (2), 495-496.

Winter, A.T., Pedersen, O.B., Rasmussen, K.V., 1981. Dislocation microstructures in fatigued copper single crystals. Acta Metall. 29, 735-748.

Zhou, D., Moosbrugger, J.C., Morrisson, D.J., 2006. Finite element simulation of PSB macroband nucleation and propagation in single crystal nickel cycled at low plastic strain amplitudes. Int. J. Plast. 22, 1336-1366. 